\title{
Identifying fabrication defects of metal packaged fibre Bragg grating sensors for smart pre-stressing strands
}

\author{
I. Mckeeman, P. Niewczas \\ Department of Engineering \\ University of Strathclyde \\ Glasgow, United Kingdom \\ iain.mckeeman@strath.ac.uk
}

\author{
M. Johnston \\ Civil Design Group \\ EDF Energy Nuclear Generation, Ltd \\ East Kilbride, United Kingdom
}

\begin{abstract}
Metal packaged fibre Bragg grating sensors have the potential to provide reliable measurements of temperature and strain in high stress environments for the purpose of structural health monitoring. However due to the induction brazing fabrication process a small percentage of sensors are found to have defective brazed joints. We demonstrate a defect identification procedure derived from the batch temperature calibration of fibre Bragg grating sensors for temperature and strain measurement, allowing defective sensors to be identified before installation. The procedure was demonstrated on a sample of twelve temperature sensors fabricated for a small-scale smart pre-stressing strand validation test.
\end{abstract}

Keywords-strain measurement; fiber Bragg gratings; condition monitoring

\section{INTRODUCTION}

Fibre optic sensors lend themselves well to remote monitoring of structures due to their excellent multiplexing capability and small size, among many other benefits [1]. Fibre Bragg gratings (FBGs) in particular are a class of their own in fibre sensing as they offer semi-distributed measurement of temperature and strain by reflecting distinct wavelengths that are strain and temperature dependent.

FBGs are fragile by nature so must be packaged to improve their mechanical strength [2]. Metal packaged FBGs offer several advantages over epoxy encapsulation, including low creep and mechanical reliability [3]. The metal packaging also provides increased structural and chemical protection making them suitable for remote sensing in harsh environments such as those in the nuclear and oil and gas industries [4], [5]. As a consequence, metal packaged FBGs meet many of the requirements for structural health monitoring (SHM) within pre-stressed concrete containment vessels (PCCVs) [6]. PCCVs rely on pre-stressed steel tendons to provide additional concrete compression to compensate against expansion due to increased pressure within the structure. Over time the pre-stress levels in the tendons reduce due to long-term and short-term effects in the concrete and steel [7]. The short term effects, such as anchor slippage and friction within the tendon ducts occur at the time of stressing. Whereas long term effects like concrete creep, shrinkage and steel relaxation can occur over years and decades. Therefore, it is important to monitor the pre-stress levels in a PCCV. Currently there are a limited number of monitoring techniques for measuring the pre-stress loss in PCCVs, these include lift off measurements and installation of load cells in ungrouted tendons [8], [9]. There is currently no reliable method for determining the loss of prestress in grouted tendons. Consequently, it would be beneficial to develop an in-situ system for monitoring the level of prestress in the steel tendons.

This work reports on further development of such a technique, in particular ensuring the sensors designed for this application are reliable and free from fabrication defects. As with many sensing applications in SHM this is a stringent constraint, as once the sensors have been deployed access to them is either impossible or severely limited.

\section{SENSORS AND THE FABRICATION PROCESS}

The sensors presented here were fabricated for a small scale test of a sensor system involving twenty four (12 strain and 12 temperature) sensors. They were attached to four steel prestressing strands to be inserted into two concrete beams for a long term assessment of the sensors performance and their practical application. The FBG encapsulation method applied has been previously documented [3], [10]. To summarise, typeI FBGs written in copper alloy coated optical fibre were induction brazed into metal capillaries. The temperature sensors were attached to the steel strands with silicon sealant at one end of the capillary so that no strain was transferred. Whilst for the strain sensors, metal shims were brazed to each end of the capillary and then spot welded to the steel strand (Fig. 1).

A high temperature brazing solder was utilised to reduce the creep rate of the sensor joints when subject to the high stress levels of this application (the steel strands are subject to $80 \%$ ultimate tensile strength of steel) [11], [12]. This ensures the sensors provide a reliable measurement under loading. However, the temperature required to melt the solder is around $650^{\circ} \mathrm{C}$. Therefore, the time duration that the fibre is exposed to this temperature requires careful control as conventional FBGs 
are thermally erased at high temperatures [13]. A sample of four sensors was monitored for reflectivity losses during the fabrication process; the average peak reflectivity loss was $35 \%$ which compares well with the previous study [3].

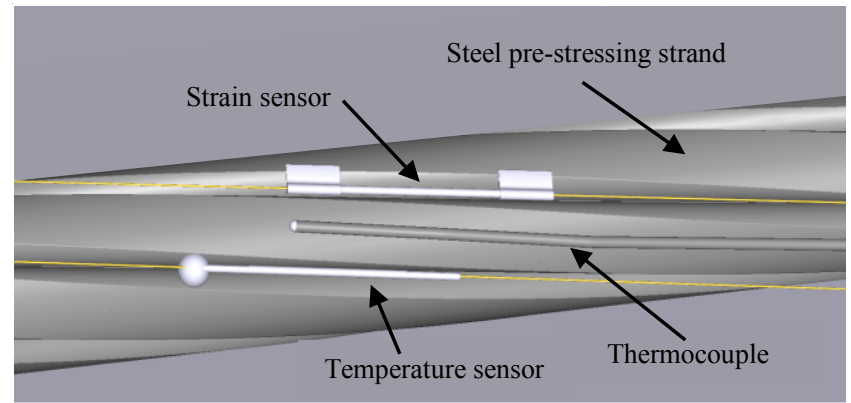

Fig. 1. A diagram of a strain (top) and temperature (bottom) FBG sensor attached to a steel pre-stressing strand. The strain sensor was spot welded to the steel strand whilst the temperature sensor was attached with silicone. A thermocouple can be seen in between the two sensors for monitoring purposes.

\section{POTENTIAL SENSOR DEFECTS}

The two main defects from the sensor fabrication process occur due to the fibre-capillary joint either being exposed to high temperature for too long; or conversely for too short a time. When the metal coated fibre is exposed to high temperature for a prolonged period, the molten brazing solder removes the metal coating of the fibre, leaving a bare exposed section at the end of the capillary. If the brazing paste does not reach the required temperature to melt all its constituent components then a brittle, porous joint is made. This does not provide a suitable bond between the capillary and the optical fibre. By encasing the sensor in resin and exposing the joints by lapping, the joints can be inspected under a microscope and these two types of fabrication defects can be identified. However, such a destructive technique renders the sensor unusable. External inspection is sufficient in most cases. However, a joint can appear free from defect externally, while internally it may be of poor quality. Furthermore, such failed sensors may appear fully operational when tested and require additional investigation to be identified as defective ${ }^{1}$. As proposed in this work, such sensors can be identified during the batch temperature calibration process as will be discussed below. Consequently, this technique will constitute a form of an environmental stress screening procedure.

For clarification, the data presented here comes from calibration of the twelve temperature sensors. However, both temperature and strain sensors follow the same initial stage of brazing an FBG into a capillary. The strain sensors contain a second brazed joint attaching two shims onto the capillary. This joint has been tested on a stressing vice and it was found that the joint was stronger than the capillary itself in all cases. Therefore, the fibre-capillary joint is the primary source of

1 It must be noted that through careful optimisation of the brazing process, brazing defects can be greatly reduced, and indeed this was confirmed whilst fabricating twenty four sensors - only $8 \%$ were found defective. But as stated previously, it is critical to ensure that $100 \%$ of the sensors are performing as expected before installation. sensor defect for both the temperature and strain sensors. The defect identification procedure can accordingly be applied to both temperature and strain sensors.

\section{DEFECT IDENTIFICATION THROUGH BATCH TEMPERATURE CALIBRATION}

\section{A. Temperature calibration}

The twelve temperature FBG sensors for the small scale test were calibrated at the same time in an environmental chamber. A J-type thermocouple was used as the temperature reference. Both the thermocouple and the FBGs were mounted on an aluminium plate. This helped to reduce temperature fluctuations between the thermocouple and FBGs due to the relatively high thermal conductivity of aluminium.

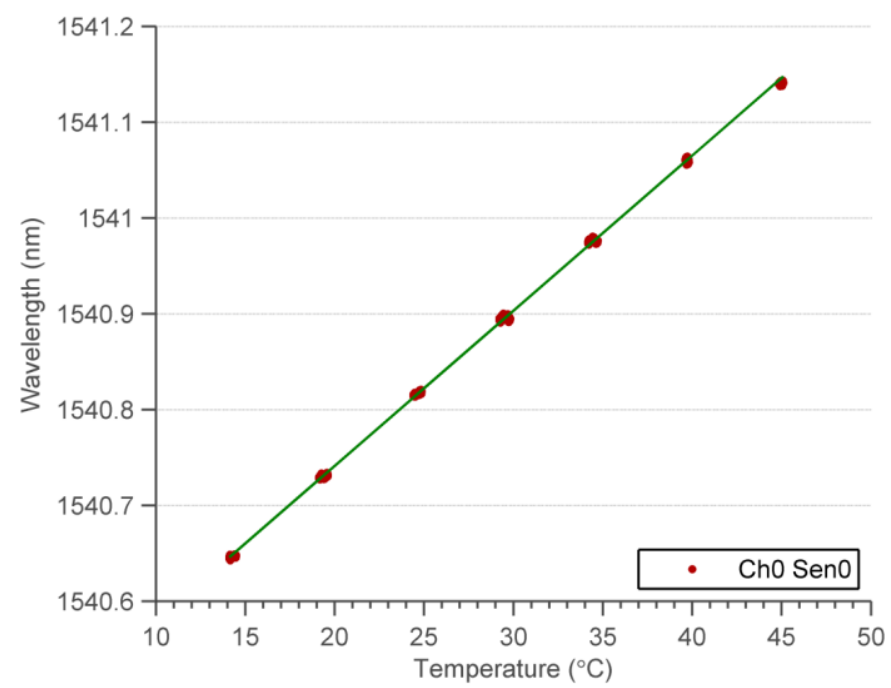

Fig. 2. An example of temperature sensor calibration (from four temperature cycles) in an environmental chamber. The data points are well placed on the least squares line of best fit.

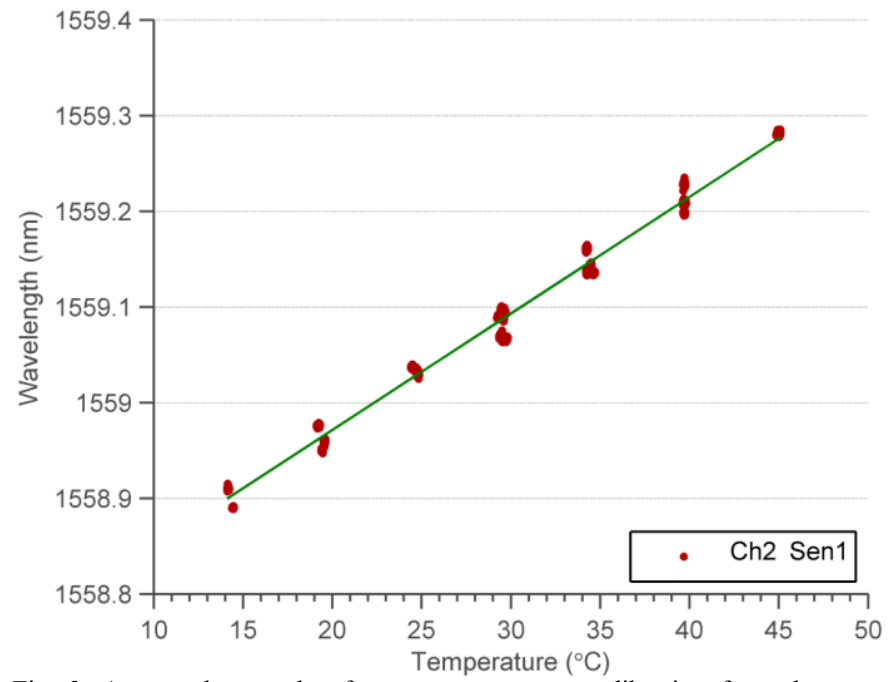

Fig. 3. A second example of temperature sensor calibration from the same batch. Here it is clear the calibration is not as repeatable as the sensor in Fig. 1. This could be due to either the calibration procedure or the sensor itself. 
The sensors were subjected to four heating cycles from 10$50^{\circ} \mathrm{C}$. The temperature was stepped in $10^{\circ} \mathrm{C}$ intervals and held at each point for two hours to allow the temperature to stabilise. Consequently, only data points from the end of the two hours of constant temperature were used for calibration. A temperature conversion coefficient $\left(\mathrm{pm} /{ }^{\circ} \mathrm{C}\right)$ was obtained from a least squares fit to the data (as seen in Fig. 2 and 3).

\section{B. Defect identification}

During temperature calibration the metal packaged sensors are heated and the metal capillaries linearly expand. The larger thermal expansion of the capillary stresses the joints between the optical fibre and the capillary. An imperfect joint between the fibre and capillary will allow their relative movement, resulting in an inconsistent temperature coefficient for the sensor. By temperature characterising multiple sensors in one run, the experimental variation between each temperature cycle can be tracked providing an indication of the repeatability of the calibration process (Fig. 4). Sensors that do not match the trend in experimental variation between cycles can be identified. As discussed above, the most likely cause of an inconsistent temperature coefficient for the sensors is a defective joint between the capillary and fibre.

\section{Identification of two defective sensors}

Fig. 2 and 3 show the plots obtained from four temperature cycles for two different temperature sensors. It is clear from the graphs that the data points obtained for sensor Ch2Sen1 lie further from the line of best fit compared to Ch0Sen0 (both graphs have the same wavelength scale for clarity). To investigate further, the temperature coefficients obtained for each individual cycle for the twelve temperature sensors were plotted on the same graph as seen in Fig. 4. Several pieces of information can be extracted. First, most of the sensors had the same magnitude of variation in their temperature coefficient between each cycle. This difference can be attributed to the experimental variation of the temperature calibration procedure. Secondly, the average temperature coefficient was in the range of $15.5-16.5 \mathrm{pm} /{ }^{\circ} \mathrm{C}$, therefore any sensors with a coefficient smaller than this should be investigated further as this suggests there was an imperfect contact between the capillary and fibre. Thirdly, there were two sensors (namely Ch2Sen1 and Ch3Sen0) which did not follow the general temperature coefficient trend of the other sensors. Only by calibrating multiple sensors in one run can a sensor be identified as unsuitable for deployment. Without this it would be hard to distinguish between experimental variation and defects in the brazed joints.

Fig. 5 shows further evidence that the two sensors identified in Fig. 4 did indeed have a defective brazed joint. The two sensors (Ch2Sen 1 and $\mathrm{Ch} 3 \mathrm{Sen} 0)$ were held at $35^{\circ} \mathrm{C}$ for approximately 8 hours. Their wavelength response (converted to temperature in the graph) clearly showed a large decrease over a short time scale. This would indicate that under stress, the fibre exiting the capillary slipped due to the tension

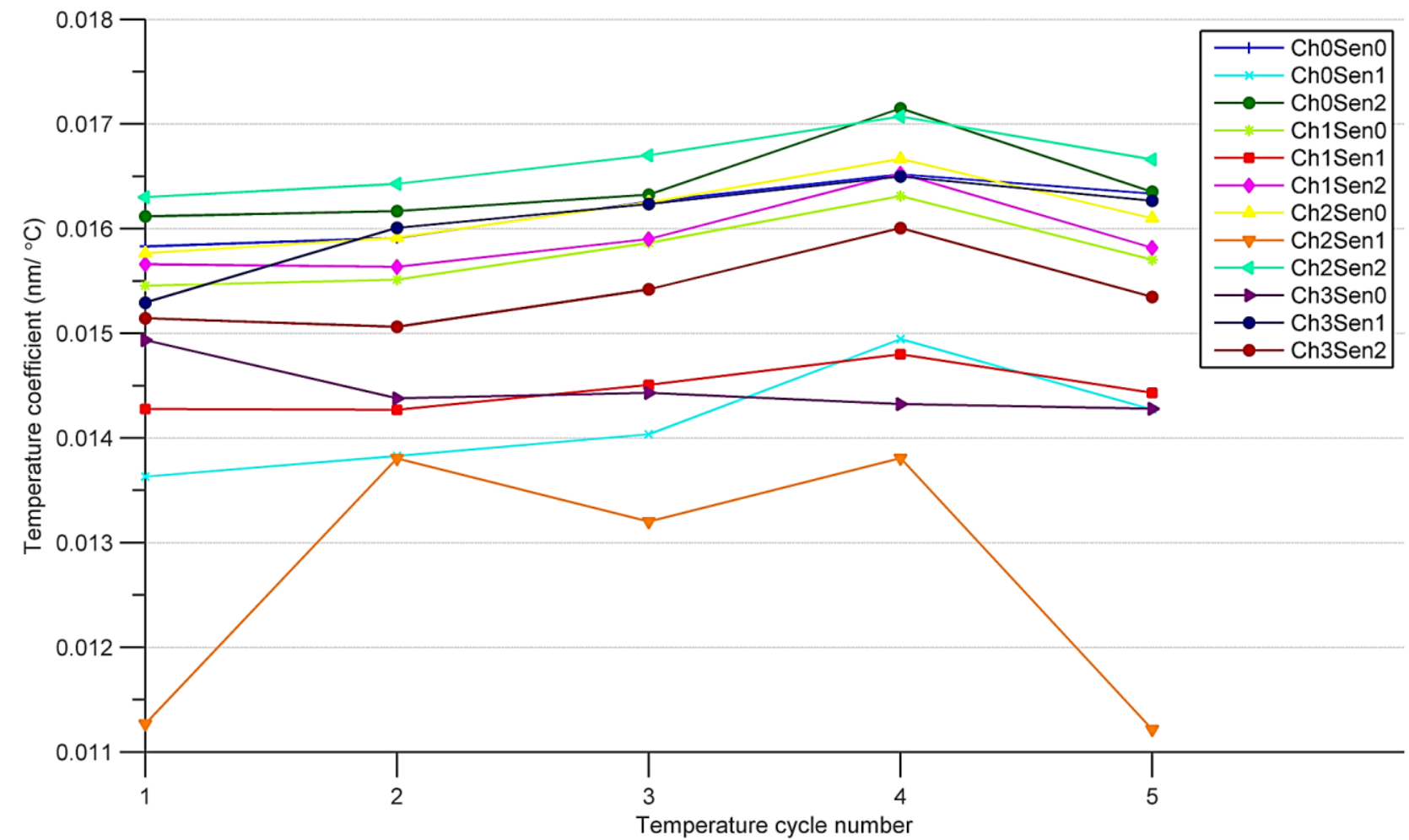

Fig. 4. Variation in the temperature coefficient of twelve sensors for five temperature calibration cycles. The twelve temperature sensors shown here were calibrated in one run, allowing the experimental variation of each cycle to be monitored by following the general trend of the majority of the sensors. Sensors with inconsistent temperature coefficients can be identified. 


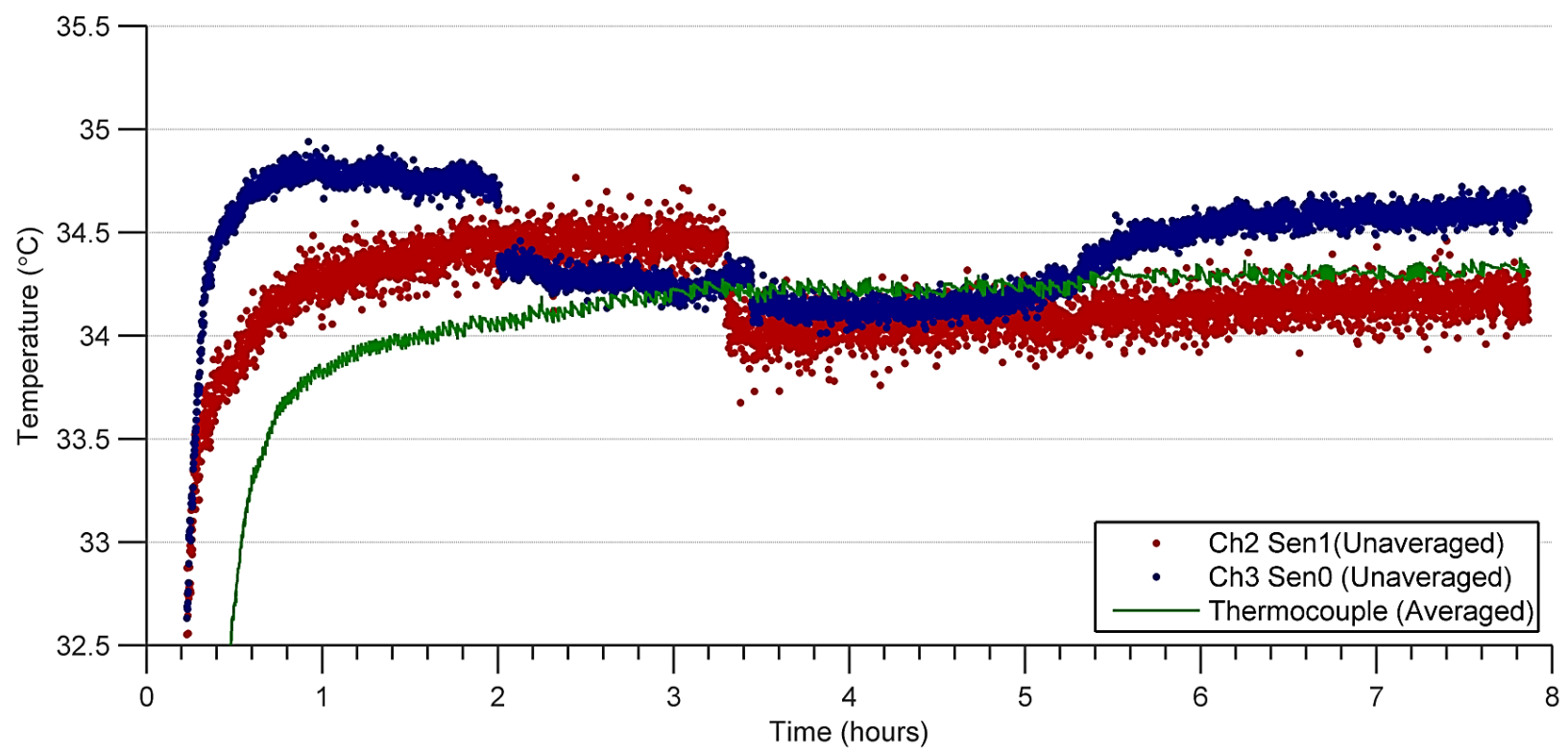

Fig. 5. The wavelength response converted to temperature of two temperature sensors held at around $35^{\circ} \mathrm{C}$ for 8 hours. A significant shift (towards shorter wavelength) was observed. This provides an indication that the fibre slipped in the capillary as the brazed joint was put under stress due to the thermal expansion of the metal capillary.

in the fibre reaching the critical magnitude needed in order to overcome the fibre-capillary bond. Sensors that were identified as functioning properly did not show the rapid decrease in wavelength seen in Fig. 5. Therefore, it is proposed that prior to calibration, sensors are held at a temperature around $60^{\circ} \mathrm{C}$ for 8 hours for a preliminary defect screening. Afterwards, the temperature calibration procedure will identify any additional sensors that do not perform consistently compared to the other sensors in the sample.

\section{CONCLUSION}

Presented in the paper is a method for identifying defects within the brazed joints of metal packaged fibre Bragg grating sensors for temperature and strain measurement. Twenty four sensors were fabricated via an induction brazing process. This was the first time a relatively large number of sensors had been fabricated allowing for a statistical sample to be investigated for defects. A defect is most likely to occur at the joint between the capillary and fibre, as a proper bond here is critical for the consistent performance of these sensors. Through optimising the brazing process, a high percentage (92\%) of consistently performing sensors were fabricated. Any defective sensors can be identified by the method proposed in this paper. It utilises a preliminary defect screening by subjecting the sensors to an elevated constant temperature for 8 hours. Following this, the repeated temperature cycles of the temperature calibration procedure can determine sensors with inconsistent temperature coefficients from cycle to cycle. This is only possible if a batch of sensors is calibrated together in order to distinguish between experimental variation in the temperature coefficient and inconsistency due to defective brazing joints. By applying the temperature coefficient variation analysis, two temperature sensors were identified as performing inconsistently. This was further confirmed when they were placed at $35^{\circ} \mathrm{C}$ for eight hours, where a significant decreasing wavelength shift over a short period occurred for both sensors, signifying that the fibre slipped within the capillary. By discovering inconsistent sensor performance before permanent attachment to the steel strands, the reliability and performance of this fibre Bragg grating temperature and strain sensing technique can be improved.

\section{ACKNOWLEDGMENT}

This work was supported by the Engineering and Physical Sciences Research Council (EPSRC) and EDF Energy Nuclear Generation, Ltd.

\section{REFERENCES}

[1] J. M. Lopez-Higuera, L. Rodriguez Cobo, A. Quintela Incera, and A. Cobo, "Fiber Optic Sensors in Structural Health Monitoring," J. Light. Technol., vol. 29, no. 4, pp. 587-608, Feb. 2011.

[2] Z. Zhou and J. Ou, "Development of FBG sensors for Structural Health Monitoring in civil infrastructures," in Sensing issues in civil structural health monitoring, Springer, 2005, pp. 197-207.

[3] M. Perry, P. Niewczas, M. Johnston, K. Cook, and J. Canning, "Induction Brazing of Type-I Fiber Bragg Gratings Into Kovar Ferrules Exploiting Curie Transition,” IEEE Sens. J., vol. 13, no. 2, pp. 816-823, Feb. 2013.

[4] M. Perry, P. Niewczas, and M. Johnston, "Effects of Neutron-Gamma Radiation on Fiber Bragg Grating Sensors: A Review," IEEE Sens. J., vol. 12, no. 11, pp. 3248-3257, Nov. 2012.

[5] S. J. Mihailov, "Fiber Bragg Grating Sensors for Harsh Environments," Sensors, vol. 12, no. 12, pp. 1898-1918, Feb. 2012.

[6] M. Perry, Z. Yan, Z. Sun, L. Zhang, P. Niewczas, and M. Johnston, "High stress monitoring of prestressing tendons in nuclear concrete vessels using fibre-optic sensors," Nucl. Eng. Des., vol. 268, pp. 35-40, Mar. 2014.

[7] E. G. Nawy, Prestressed concrete A fundamental approach, 5th ed. New Jersey, USA: Prentice Hall, 2010. 
[8] P. Anderson, "Thirty years of measured prestress at Swedish nuclear reactor containments," Nucl. Eng. Des., vol. 235, no. 21, pp. 2323-2336, Oct. 2005.

[9] L. M. Smith, "In-service monitoring of nuclear-safety-related structures," Struct. Eng., vol. 74, no. 12, pp. 210-211, Jun. 1996.

[10] G. Fusiek, M. Perry, P. Niewczas, and M. Johnston, "Deformation monitoring in prestessing tendons using fibre Bragg gratings encapsulated in metallic packages," in SPIE 9157, 23rd International Conference on Optical Fibre Sensors, 2014, vol. 9157A6.

[11] Z. Mei and J. W. Morris, "Superplastic creep of low melting point solder joints,” J. Electron. Mater., vol. 21, no. 4, pp. 401-407, Apr. 1992.

[12] Q. Tan and Y. C. Lee, "Soldering technology for optoelectronic packaging," in Electronic Components and Technology Conference, 1996. Proceedings., 46th, 1996, pp. 26-36.

[13] S. R. Baker, H. N. Rourke, V. Baker, and D. Goodchild, "Thermal decay of fiber Bragg gratings written in boron and germanium codoped silica fiber,” Light. Technol. J. Of, vol. 15, no. 8, pp. 1470-1477, 1997. 